\title{
Inorganic Acid-Catalyzed Tautomerization of Vinyl Alcohol to Acetaldehyde
}

\author{
Amir Karton* \\ School of Chemistry and Biochemistry, The University of Western Australia, Perth, WA \\ 6009, Australia.
}

\begin{abstract}
The vinyl alcohol-acetaldehyde tautomerization reaction has recently received considerable attention as a potential route for the formation of organic acids in the troposphere [Andrews et al. Science 2012, 337, 1203]. We examine the catalytic effect of inorganic acids in the troposphere (e.g. $\mathrm{HNO}_{3}, \mathrm{H}_{2} \mathrm{SO}_{4}$ and $\mathrm{HClO}_{4}$ ) on the vinyl alcoholacetaldehyde tautomerization reaction, by means high-level thermochemical procedures. We show that $\mathrm{H}_{2} \mathrm{SO}_{4}$ and $\mathrm{HClO}_{4}$ catalysts lead to near-zero reaction barrier heights for the vinyl alcohol $\rightarrow$ acetaldehyde reaction, and to low reaction barrier heights in the reverse direction $\left(\Delta H_{298}^{\ddagger}=40.6\right.$ and $39.5 \mathrm{~kJ} \mathrm{~mol}^{-1}$, respectively $)$.
\end{abstract}

Keywords: Atmospheric chemistry $\bullet$ keto-enol tautomerization $\bullet$ sulfuric acid $\bullet$ perchloric acid $\bullet$ homogeneous catalysis $\bullet$ computational thermochemistry $\bullet$ W1-F12 theory

Cite as:

A. Karton. Chem. Phys. Lett. 592, 330-333 (2014).

http://dx.doi.org/10.1016/j.cplett.2013.12.062 


\section{INTRODUCTION}

Small carboxylic acids are the most abundant organic acids in the troposphere and play a key role in acid deposition. ${ }^{1,2,3,4,5}$ Despite their abundance, current kinetic models are not able to accurately predict their formation in both the urban and rural troposphere, implying a missing secondary source for carboxylic acids. ${ }^{1}$ Shallcross and co-workers, showed that oxidation of vinyl alcohol significantly enhances the formation of formic acid in the troposphere, and suggest that enolic species may be the missing secondary source of carboxylic acids. ${ }^{2}$ Recently, Jordan, Kable and co-workers proposed that photo-induced tautomerization of acetaldehyde to vinyl alcohol is a potential route for the formation of vinyl alcohol in the troposphere. ${ }^{6,7,8}$

The uncatalyzed keto-enol tautomerization reaction has a high barrier and is not a viable route under tropospheric conditions $\left(\Delta H_{298}^{\ddagger}=236.1 \mathrm{~kJ} \mathrm{~mol}^{-1}\right.$, relative to the free vinyl alcohol, vide infra) ${ }^{7,9,10}$ In the present Letter, we examine the catalytic effect of species that are of atmospheric importance on the vinyl alcohol $\leftrightarrow$ acetaldehyde tautomerization reaction. High-level ab initio calculations (with the W1-F12 thermochemical protocol) ${ }^{11}$ were performed in the gas-phase in order to explore the uncatalyzed and catalyzed potential energy surfaces (Computational Methods Section). We consider the following catalysts: sulfuric acid $\left(\mathrm{H}_{2} \mathrm{SO}_{4}\right)$, perchloric acid $\left(\mathrm{HClO}_{4}\right)$, phosphoric acid $\left(\mathrm{H}_{3} \mathrm{PO}_{4}\right)$, carbonic acid $\left(\mathrm{H}_{2} \mathrm{CO}_{3}\right)$, formic acid $(\mathrm{HC}(\mathrm{O}) \mathrm{OH})$, nitric acid $\left(\mathrm{HNO}_{3}\right)$, hydroperoxyl radical $\left(\mathrm{HO}_{2}\right)$ and water $\left(\mathrm{H}_{2} \mathrm{O}\right)$. We show that a double proton shift between the catalyst and the substrate can lead to energetically lowlying transition structures (TSs) for the gas-phase tautomerization reaction. In particular, the $\mathrm{H}_{2} \mathrm{SO}_{4}$ and $\mathrm{HClO}_{4}$ catalysts lead to submerged barrier heights on the enthalpic surface at 298 $\mathrm{K}\left(\Delta H_{298}^{\ddagger}\right)$ (relative to the free vinyl alcohol), and to low reaction barrier heights of 40.6 and $39.5 \mathrm{~kJ} \mathrm{~mol}^{-1}$, respectively (relative to the free acetaldehyde). These findings may have significant consequences for current atmospheric chemical models since mineral acids (e.g. $\mathrm{H}_{2} \mathrm{SO}_{4}$ and $\mathrm{HNO}_{3}$ ) and the hydroperoxy radical are prominent species in the troposphere. ${ }^{3,12,13,14,15,16}$

\section{COMPUTATIONAL METHODS}

High-level ab initio calculations were performed in order to explore the potential energy surface for the uncatalyzed and catalyzed tautomerization reaction. The W1 thermochemical protocol ${ }^{17}$ (and its explicitly-correlated W1-F12 version) ${ }^{11}$ are widely used for the calculation of thermochemical and kinetic properties. ${ }^{18,19}$ These theories represent a layered extrapolation to the relativistic, all-electron $\operatorname{CCSD}(\mathrm{T})$ (coupled cluster with singles, 
doubles, and quasiperturbative triple excitations) basis-set-limit energy, and can achieve "sub-chemical accuracy" (e.g., they are associated with a mean absolute deviation from accurate atomization energies of $2.0 \mathrm{~kJ} \mathrm{~mol}^{-1}$ ) for molecules whose wave functions are dominated by dynamical correlation. ${ }^{11,17,18,19,20,21,22,23}$

The geometries of all structures have been obtained at the B3LYP-D3/A'VTZ level of theory, ${ }^{24,25}$ where $\mathrm{A}^{\prime} \mathrm{V} n \mathrm{Z}$ indicates the combination of the standard correlation-consistent cc$\mathrm{pV} n \mathrm{Z}$ basis sets on $\mathrm{H}^{26}$ the aug-cc-pVnZ basis sets on first-row elements, ${ }^{27}$ and the aug-cc$\mathrm{pV}(n+\mathrm{d}) \mathrm{Z}$ basis sets on second-row elements. ${ }^{28}$ Harmonic vibrational analyses have been performed to confirm each stationary point as either an equilibrium structure (i.e., all real frequencies) or a transition structure (TS) (i.e., with one imaginary frequency). Zero-point vibrational energy (ZPVE) and enthalpic corrections have been obtained from such calculations. The connectivities of the transition structures were confirmed by performing intrinsic reaction coordinate (IRC) calculations. ${ }^{29}$ All geometry optimizations and frequency calculations were performed using the Gaussian 09 program suite. ${ }^{30}$

In order to obtain reliable reaction energies and barrier heights for the uncatalyzed and catalyzed reactions, calculations have been carried out using the high-level, ab initio W1-F12 and W1 procedures with the Molpro 2012.1 program suite. ${ }^{31} \mathrm{~W} 1-\mathrm{F} 12$ combines explicitlycorrelated F12 methods $^{32,33}$ with extrapolation techniques in order to approximate the $\operatorname{CCSD}(\mathrm{T})$ basis-set-limit energy. Due to the drastically accelerated basis-set convergence of the F12 methods, ${ }^{34,35} \mathrm{~W} 1-\mathrm{F} 12$ is superior to the original W1 method not only in terms of performance but also in terms of computational cost. ${ }^{11}$ The computational protocol of the W1-F12 method has been specified and rationalized in detail in ref. 11. In brief, the HartreeFock component is extrapolated from the VDZ-F12 and VTZ-F12 basis sets, using the $\mathrm{E}(L)=$ $\mathrm{E}_{\infty}+\mathrm{A} / L^{\alpha}$ two-point extrapolation formula, with $\alpha=5$ (where VnZ-F12 denotes the ccpVnZ-F12 basis sets of Peterson et al., ${ }^{34}$ which were specifically developed for explicitly correlated calculations). The valence CCSD-F12 correlation energy is extrapolated from the same basis sets, using the same two-point extrapolation formula but with $\alpha=3.67$. In all of the explicitly-correlated coupled cluster calculations the diagonal, fixed-amplitude 3C(FIX) ansatz $^{36,37}$ and the CCSD-F12b approximation are employed. ${ }^{38}$ The $(\mathrm{T})$ valence correlation energy is obtained in the same way as in the original W1 theory, ${ }^{17}$ i.e., extrapolated from the $\mathrm{A}^{\prime} \mathrm{VDZ}$ and $\mathrm{A}^{\prime} \mathrm{VTZ}$ basis sets using the above two-point extrapolation formula with $\alpha=3.22$. The CCSD inner-shell contribution is calculated with the core-valence weighted correlationconsistent aug'-cc-pwCVTZ basis set of Peterson and Dunning, ${ }^{39}$ whilst the (T) inner-shell contribution is calculated with the cc-pwCVTZ(no f) basis set (where cc-pwCVTZ(no f) 
indicates the cc-pwCVTZ basis set without the $\mathrm{f}$ functions). The scalar relativistic contribution (in the second-order Douglas-Kroll-Hess approximation) ${ }^{40,41}$ is obtained as the difference between non-relativistic CCSD(T)/A'VDZ and relativistic CCSD(T)/A'VDZ-DK calculations. $^{42}$ The diagonal Born-Oppenheimer corrections (DBOC) are calculated at the HF/A'VTZ level of theory.

\section{RESULTS AND DISCUSSION}

It is well known that the presence of one (or more) water molecules can significantly enhance the reaction rates of prototropic tautomerizations in the gas phase relative to the rates of the uncatalyzed reactions. ${ }^{9,12,43,44,45}$ For the vinyl alcohol $\rightarrow$ acetaldehyde tautomerization reaction one water catalyst reduces the barrier by more than $50 \%$. Specifically, at the W1F12 level, the following barriers $\left(\Delta H^{*} 298\right)$ are obtained: 236.1 (uncatalyzed) and $104.4\left(\mathrm{H}_{2} \mathrm{O}\right.$ catalyst) $\mathrm{kJ} \mathrm{mol}^{-1}$. Figure $1 \mathrm{a}-\mathrm{b}$ shows the TSs for the uncatalyzed and catalyzed reactions. In part, the barrier reductions may be attributed to the reduced strain energy in the TSs, as the addition of a water molecule allows for the formation of a six-membered-ring TS compared with the four-membered-ring TS in the uncatalyzed reaction. ${ }^{44,45}$ 
(a)

(e)

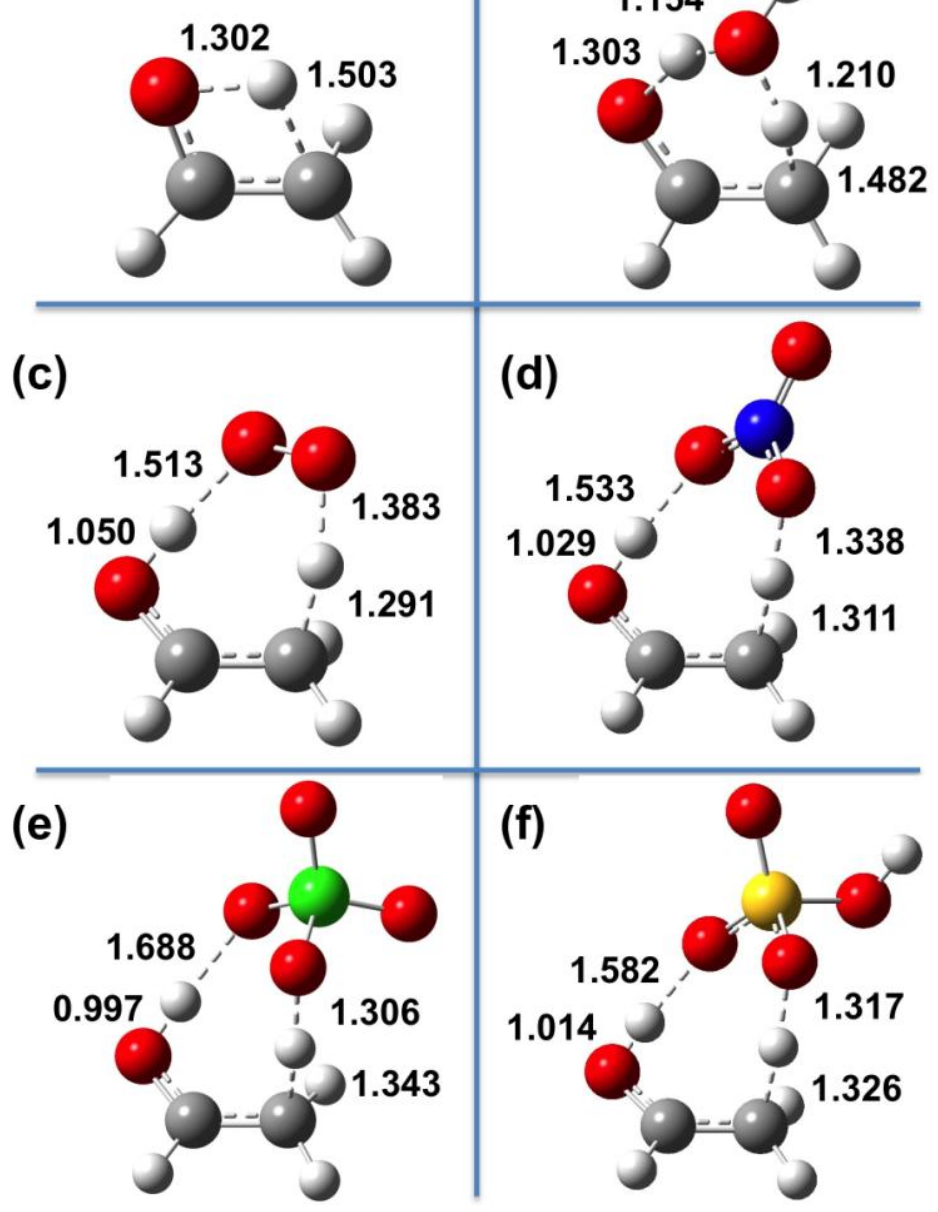

(b)

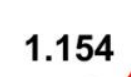

Figure 1. B3LYP-D3/A'VTZ optimized transition structures for the vinyl alcohol $\leftrightarrow$ acetaldehyde tautomerization reaction: (a) uncatalyzed; and catalyzed by: (b) $\mathrm{H}_{2} \mathrm{O}$, (c) $\mathrm{HO}_{2}$, (d) $\mathrm{HNO}_{3}$, (e) $\mathrm{HClO}_{4}$ and (f) $\mathrm{H}_{2} \mathrm{SO}_{4}$. The bonds being broken and formed in the transition structures are represented by single dashed lines, and the lengths of these bonds are given in $\AA$. Atomic color scheme: H, white; C, gray; N, blue; $\mathrm{O}$, red; $\mathrm{S}$, yellow; $\mathrm{Cl}$, green.

Recently, da Silva found that a formic acid catalyst $(\mathrm{HC}(\mathrm{O}) \mathrm{OH})$ significantly enhances the rate of the vinyl alcohol $\leftrightarrow$ acetaldehyde tautomerization reaction. ${ }^{9} \mathrm{He}$ also pointed out that other catalysts in which a carbonyl oxygen and a hydroxyl group are bound to a common atom are expected to posses catalytic properties. Using the composite G3SX procedure, ${ }^{4647}$ he calculated an enthalpic barrier $\left(\Delta H^{\ddagger}{ }_{298}\right)$ of $236.8 \mathrm{~kJ} \mathrm{~mol}^{-1}$ for the uncatalyzed tautomerization, and a barrier of $23.4 \mathrm{~kJ} \mathrm{~mol}^{-1}$ for the reaction catalyzed by a formic acid catalyst. Table 1 gives the W1-F12 enthalpies at $298 \mathrm{~K}\left(\Delta H_{298}\right)$ for the reactant complexes (RC), transition structures (TS), and product complexes (PC) located along the catalyzed pathways for the vinyl alcohol $\rightarrow$ acetaldehyde reaction. A schematic representation of the enthalpy energy profile is given in Figure 2. For the uncatalyzed 
reaction we obtain a barrier of $236.1 \mathrm{~kJ} \mathrm{~mol}^{-1}$, which is in excellent agreement with the reaction barrier of da Silva. ${ }^{9}$ An $\mathrm{HO}_{2}$ radical catalyst reduces the barrier by more than $80 \%$ $\left(\Delta H_{298}^{\star}=46.6 \mathrm{~kJ} \mathrm{~mol}^{-1}\right)$. In part, $\mathrm{HO}_{2}$ is a more effective catalyst than $\mathrm{H}_{2} \mathrm{O}$ due to the reduced strain energy associated with a seven-membered-ring $\mathrm{TS}\left(\mathrm{HO}_{2}\right.$ catalyst, Figure 1c) relative to a six-membered-ring $\mathrm{TS}\left(\mathrm{H}_{2} \mathrm{O}\right.$ catalyst, Figure $\left.1 \mathrm{~b}\right)$. This is demonstrated by angles between the $\mathrm{O}-\mathrm{C}-\mathrm{C}$ backbone of the substrate and the protons that are being transferred. Specifically, the $\angle \mathrm{C}-\mathrm{C} \cdots \cdot \mathrm{H}$ angles are $66.8^{\circ}$ (uncatalyzed TS, Figure 1a), $91.8^{\circ}\left(\mathrm{H}_{2} \mathrm{O}\right.$ catalyst, Figure $1 \mathrm{~b})$ and $102.8^{\circ}\left(\mathrm{HO}_{2}\right.$ catalyst, Figure $\left.1 \mathrm{c}\right)$, and the $\angle \mathrm{C}-\mathrm{O} \bullet \bullet \cdot \mathrm{H}$ angles are $76.9^{\circ}$ (uncatalyzed TS), $102.5^{\circ}\left(\mathrm{H}_{2} \mathrm{O}\right.$ catalyst) and $108.5^{\circ}\left(\mathrm{HO}_{2}\right.$ catalyst). A formic acid catalyst results in a W1-F12 reaction barrier of $26.2 \mathrm{~kJ} \mathrm{~mol}^{-1}$. We note that the G3SX barrier of da Silva $^{9}\left(23.4 \mathrm{~kJ} \mathrm{~mol}^{-1}\right)$ is in good agreement with our benchmark W1-F12 barrier, in accordance with the expected accuracy of the more approximate G3SX procedure for reaction barrier heights. ${ }^{11,44,45,48,49}$ The reaction barrier obtained with the formic acid catalyst is lower by $20.4 \mathrm{~kJ} \mathrm{~mol}^{-1}$ than the one obtained with the $\mathrm{HO}_{2}$ catalyst (Table 1). Again, this may be attributed in part to the reduction in strain energy when moving from a sevenmembered-ring TS to an eight-membered-ring TS.

Table 1. Enthalpies at $298 \mathrm{~K}\left(\Delta H_{298}, \mathrm{~W} 1-\mathrm{F} 12, \mathrm{~kJ} \mathrm{~mol}^{-1}\right)$ for the reactant complexes (RC), transition structures (TS), and product complexes (PC) located on the potential energy surface for the uncatalyzed and catalyzed vinyl alcohol $\rightarrow$ acetaldehyde reaction. ${ }^{a}$

\begin{tabular}{lccc} 
Catalyst & RC & TS & PC \\
Uncat $^{b}$ & & 236.1 & \\
$\mathrm{H}_{2} \mathrm{O}$ & -20.1 & 104.4 & -60.8 \\
$\mathrm{HO}_{2}$ & -26.1 & 46.6 & -77.4 \\
$\mathrm{HNO}_{3}$ & -27.0 & 30.9 & -79.4 \\
$\mathrm{HC}(\mathrm{O}) \mathrm{OH}$ & -33.2 & 26.2 & -73.9 \\
$\mathrm{H}_{2} \mathrm{CO}_{3}$ & -31.5 & 22.9 & -77.2 \\
$\mathrm{H}_{3} \mathrm{PO}_{4}$ & -46.2 & 0.2 & -82.4 \\
$\mathrm{HClO}_{4}$ & -34.6 & -0.5 & -92.9 \\
$\mathrm{H}_{2} \mathrm{SO}_{4}$ & -39.6 & -1.6 & -89.3 \\
${ }^{a} \Delta H_{298}$ values are calculated relative to the \\
free vinyl alcohol and catalyst; the reaction \\
enthalpy is $-41.1 \mathrm{~kJ}$ mol ${ }^{-1}$. \\
${ }^{b}$ Energy barrier for the uncatalyzed reaction. \\
\hline
\end{tabular}




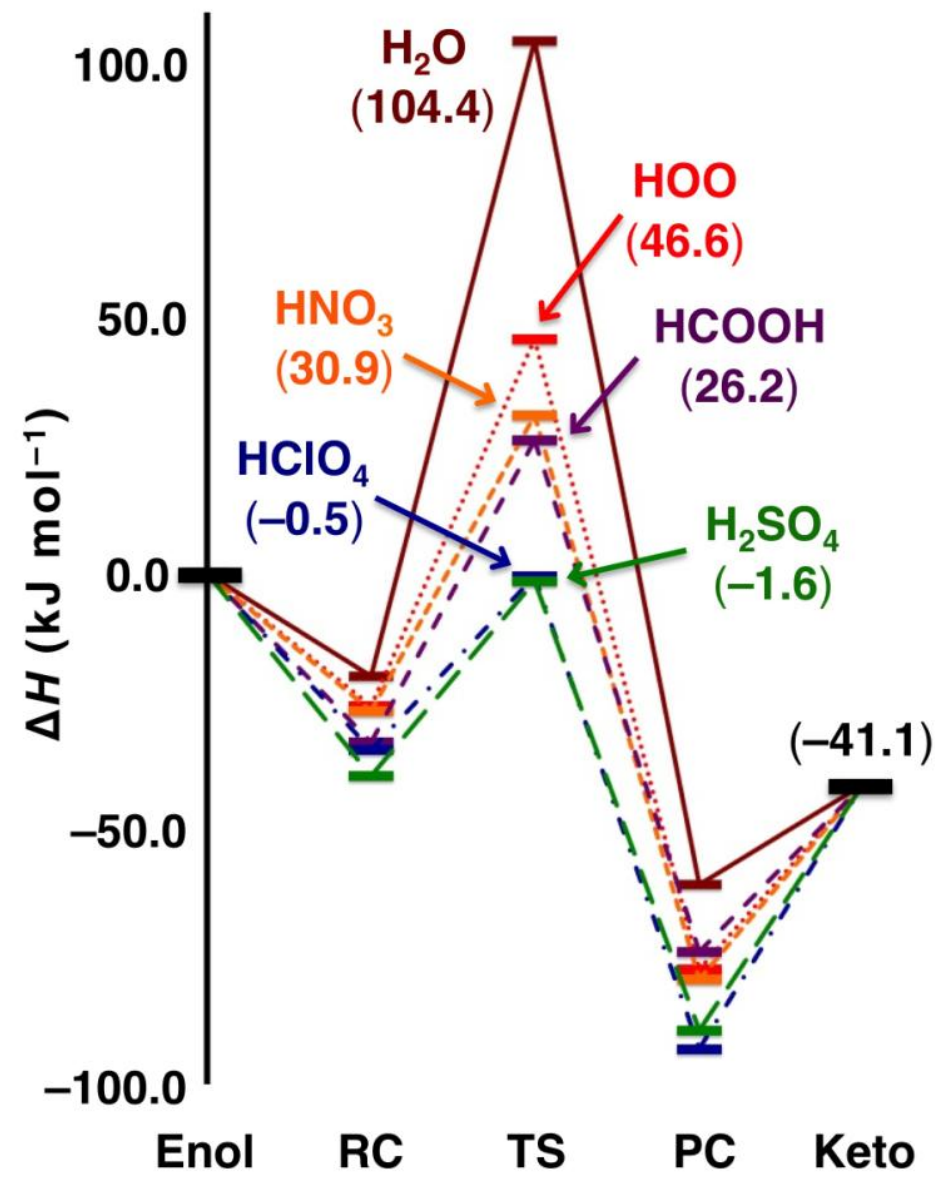

Figure 2. Schematic potential energy profiles for the vinyl alcohol $\rightarrow$ acetaldehyde reaction catalyzed by $\mathrm{H}_{2} \mathrm{O}, \mathrm{HO}_{2}, \mathrm{HNO}_{3}, \mathrm{H}_{2} \mathrm{CO}_{3}, \mathrm{HClO}_{4}$ and $\mathrm{H}_{2} \mathrm{SO}_{4}\left(\Delta \mathrm{H}_{298}\right.$, W1-F12, $\left.\mathrm{kJ} \mathrm{mol}^{-1}\right)$. The energies of the reactant complexes (RC) and product complexes (PC) are given in Table 1.

We turn now to the catalytic activity of the inorganic acids $\left(\mathrm{HNO}_{3}, \mathrm{H}_{2} \mathrm{CO}_{3}, \mathrm{H}_{3} \mathrm{PO}_{4}\right.$, $\mathrm{HClO}_{4}$ and $\mathrm{H}_{2} \mathrm{SO}_{4}$ ). Figure 1d-f shows the TSs for the $\mathrm{HNO}_{3}, \mathrm{HClO}_{4}$ and $\mathrm{H}_{2} \mathrm{SO}_{4}$ catalysts, while the reaction barrier heights are given in Table 1 (and Figure 2). A common structural motif shared by all the considered acids is that the central atom $\mathrm{X}(\mathrm{X}=\mathrm{C}, \mathrm{N}, \mathrm{P}, \mathrm{S}$ and $\mathrm{Cl})$ connects an $\mathrm{X}=\mathrm{O}$ and an $\mathrm{X}-\mathrm{OH}$ groups (Scheme 1). The acids in which $\mathrm{X}$ is a first-row element $\left(\mathrm{H}_{2} \mathrm{CO}_{3}\right.$ and $\left.\mathrm{HNO}_{3}\right)$ result in reaction barriers of 22.9 and $30.9 \mathrm{~kJ} \mathrm{~mol}^{-1}$, respectively. Whereas oxoacid catalysts in which $\mathrm{X}$ is a second-row element result in submerged (or nearly submerged) reaction barrier heights (relative to the free vinyl alcohol and catalyst). Specifically, at the W1-F12 level, we obtain reaction barriers of $0.2\left(\mathrm{H}_{3} \mathrm{PO}_{4}\right)$, $0.5\left(\mathrm{HClO}_{4}\right)$ and $-1.6\left(\mathrm{H}_{2} \mathrm{SO}_{4}\right) \mathrm{kJ} \mathrm{mol}^{-1}$. Therefore, in the presence of a second-row oxoacid, the catalytic conversion of vinyl alcohol to acetaldehyde should proceed even at low temperatures. 
It is evident that the nature of the central atom of the oxoacid plays an important role in the catalytic efficiency. This may be attributed in part to the reduced strain energy in the TSs when moving from a first-row to a larger second-row $\mathrm{X}$ center. Table 2 gives selected bond distances for the oxoacids considered (Scheme 1 shows the atom labeling scheme). We refer to the oxygen of the acid that exchanges a proton with the keto group as $\mathrm{O}_{\mathrm{a}}$, and to the oxygen that exchanges a proton with the methyl group as $\mathrm{O}_{\mathrm{b}}$. Inspection of Table 2 reveals that the $\mathrm{X}-\mathrm{O}_{\mathrm{a}}$ distances range between $1.248-1.268 \AA$ for the first-row acids, and between $1.462-1.499 \AA$ for the second-row acids. Similarly, the $\mathrm{X}-\mathrm{O}_{\mathrm{b}}$ distances range between 1.266$1.302 \AA$ (first-row acids) and between 1.504-1.534 $\AA$ (second-row acids). The longer X-O bonds in the second-row oxoacids allow for less strained TSs. This is also demonstrated by longer $\mathrm{O}_{\mathrm{a}} \cdots \mathrm{O}_{\mathrm{b}}$ distances for the second-row oxoacids (Table 2).

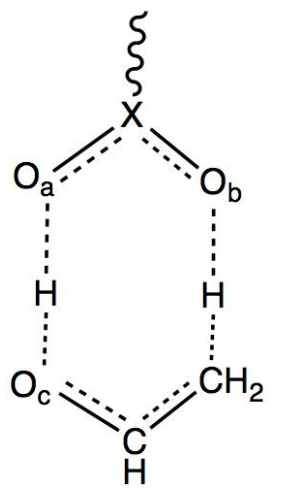

Scheme 1. Schematic representation of the TSs for the vinyl alcohol $\leftrightarrow$ acetaldehyde tautomerization reaction catalyzed by an oxoacid $\left(\mathrm{HNO}_{3}, \mathrm{H}_{2} \mathrm{CO}_{3}, \mathrm{H}_{3} \mathrm{PO}_{4}, \mathrm{HClO}_{4}\right.$ and $\left.\mathrm{H}_{2} \mathrm{SO}_{4}\right)$ with a central atom $\mathrm{X}(\mathrm{X}=\mathrm{C}, \mathrm{N}, \mathrm{P}, \mathrm{S}, \mathrm{Cl})$. The wavy bond represents additional groups attached to the $\mathrm{X}$ atom.

Table 2. Selected bond distances characterizing the oxoacid catalyst in the transition structures and in the free oxoacid (atom numbers are shown in Scheme 1, distances are given in $\AA$ ).

\begin{tabular}{lccccc} 
& \multicolumn{2}{c}{ Catalyzed TS } & \multicolumn{2}{c}{ Free oxoacid } \\
\multicolumn{1}{c}{ Catalyst } & $\mathbf{X}-\mathbf{O}_{\mathbf{a}}$ & $\mathbf{X}-\mathbf{O}_{\mathbf{b}}$ & $\mathbf{O}_{\mathbf{a}} \cdots \mathbf{O}_{\mathbf{b}}$ & $\mathbf{X}=\mathbf{O}$ & $\mathbf{X}-\mathbf{O H}$ \\
$\mathrm{HNO}_{3}$ & 1.268 & 1.302 & 2.207 & 1.208 & 1.415 \\
$\mathrm{HC}(\mathrm{O}) \mathrm{OH}$ & 1.254 & 1.266 & 2.257 & 1.198 & 1.345 \\
$\mathrm{H}_{2} \mathrm{CO}_{3}$ & 1.248 & 1.276 & 2.261 & 1.204 & 1.340 \\
$\mathrm{H}_{3} \mathrm{PO}_{4}$ & 1.499 & 1.527 & 2.455 & 1.464 & 1.592 \\
$\mathrm{HClO}_{4}$ & 1.468 & 1.534 & 2.405 & 1.423 & 1.663 \\
$\mathrm{H}_{2} \mathrm{SO}_{4}$ & 1.462 & 1.504 & 2.434 & 1.422 & 1.597 \\
\hline
\end{tabular}




\section{CONCLUSIONS}

On the basis of high-level computational modeling, we show that inorganic acids, which are ubiquitous in the troposphere can efficiently catalyze the vinyl alcohol $\leftrightarrow$ acetaldehyde tautomerization reaction. Specifically, oxoacids with a first-row center $\left(\mathrm{H}_{2} \mathrm{CO}_{3}\right.$, $\mathrm{HC}(\mathrm{O}) \mathrm{OH}$ and $\mathrm{HNO}_{3}$ ) result in barrier heights $\left(\Delta H_{298}^{*}\right)$ of $22.9-30.9 \mathrm{~kJ} \mathrm{~mol}^{-1}$ for the forward reaction (vinyl alcohol $\rightarrow$ acetaldehyde), and $64.0-72.0 \mathrm{~kJ} \mathrm{~mol}^{-1}$ for the reverse reaction (acetaldehyde $\rightarrow$ vinyl alcohol). Remarkably, oxoacid catalysts with a second-row central atom $\left(\mathrm{H}_{2} \mathrm{SO}_{4}, \mathrm{HClO}_{4}\right.$ and $\left.\mathrm{H}_{3} \mathrm{PO}_{4}\right)$ lead to submerged (or nearly submerged) barrier heights for the forward reaction, and to very low barrier heights (of $\Delta H^{\ddagger} 298=39.5-41.3 \mathrm{~kJ} \mathrm{~mol}^{-1}$ ) for the reverse reaction. Thus, the presence of these catalysts in the troposphere may enable the vinyl alcohol $\rightarrow$ acetaldehyde conversion to proceed under tropospheric conditions. For comparison, the barriers for the uncatlyzed reaction are $236.1 \mathrm{~kJ} \mathrm{~mol}^{-1}$ (vinyl alcohol $\rightarrow$ acetaldehyde) and $277.2 \mathrm{~kJ} \mathrm{~mol}^{-1}$ (acetaldehyde $\rightarrow$ vinyl alcohol). These findings may have significant consequences for current atmospheric chemical models since mineral acids such as $\mathrm{H}_{2} \mathrm{SO}_{4}$ and $\mathrm{HNO}_{3}$ are prominent species in the troposphere.

\section{Acknowledgments}

We gratefully acknowledge funding from the Australian Research Council (ARC Discovery Project Grant: DP110102336) and the generous allocation of computing time from the National Computational Infrastructure (NCI) National Facility and the support of iVEC through the use of advanced computing resources located at iVEC@UWA.

\section{Appendix A. Supplementary data}

A schematic representation of the B3LYP-D3/A'VTZ optimized TSs for the vinyl alcohol $\leftrightarrow$ acetaldehyde tautomerization reaction catalyzed by: $\mathrm{HC}(\mathrm{O}) \mathrm{OH}, \mathrm{H}_{2} \mathrm{CO}_{3}$ and $\mathrm{H}_{3} \mathrm{PO}_{4}$ (Figure S1). Component breakdown of the final $\mathrm{W} 1-\mathrm{F} 12$ reaction energies and barrier heights at $0 \mathrm{~K}$ and at $298 \mathrm{~K}$ (Table S1); Absolute energies used for obtaining the final W1-F12 reaction energies and barrier heights (Table S2); Component breakdown of the final W1 reaction energies and barrier heights (Table S3); Comparison between HF*/cc-pV\{D,T\}Z-F12 (W1F12 theory), $\mathrm{HF} / \mathrm{A}^{\prime} \mathrm{V}\{\mathrm{Q}, 5\} \mathrm{Z}$ (W2.2 theory) and $\mathrm{HF} / \mathrm{A}^{\prime} \mathrm{V}\{5,6\} \mathrm{Z}$ (W4 theory) components for the second-row systems exhibiting various degrees of inner polarization (Table S4); Comparison between the barrier heights and energies for the reactions considered in the present work obtained with the G4 and W1-F12 protocols (Table S5); W1-F12 Gibbs free 
energies at $298 \mathrm{~K}$ for the reactant complexes, transition structures, and product complexes located on the potential energy surface for the uncatalyzed and catalyzed vinyl alcohol $\rightarrow$ acetaldehyde reaction (Table S6); B3LYP-D3/A'VTZ optimized geometries for the species considered in the present work (Table S7); and full references for ref 30 (Gaussian 09) and ref 31 (Molpro 2012). Supplementary data associated with this article can be found, in the online version, at http://dx.doi.org/.

\section{Corresponding author}

*E-Mail: amir.karton@uwa.edu.au

\section{References}

1 Paulot, F.; Wunch, D.; Crounse, J. D.; Toon, G. C.; Millet, D. B.; DeCarlo, P. F.; Vigouroux, C.; Deutscher, N. M.; Gonza'lez Abad, G.; Notholt, J. et al. Atmos. Chem. Phys. 2011, 11, 1989.

2 Archibald, A. T.; McGillen, M. R.; Taatjes, C. A.; Percival, C. J.; Shallcross, D. E. Geophys. Res. Lett. 2007, 34, L21801.

${ }^{3}$ Finlayson-Pitts, B. J.; Pitts, J. N. Jr. Acid deposition: formation and fates of inorganic and organic acids in the troposphere. In Chemistry of the upper and lower atmosphere (eds. Finlayson-Pitts, B. J. and Pitts, J. N. Jr.), Academic Press, New York, 2000.

${ }^{4}$ Baboukas, E. D.; Kanakidou, M.; Mihalopoulos, N. J. Geophys. Res. 2000, 105, 14459.

${ }^{5}$ Chebbi, A.; Carlier, P. Atmos. Environ. 1996, 30, 4233.

${ }^{6}$ Heazlewood, B. R.; Maccarone, A. T.; Andrews, D. U.; Osborn, D. L.; Harding, L. B.; Klippenstein, S. J.; Jordan, M. J. T.; Kable, S. H. Nat. Chem. 2011, 3, 443.

${ }^{7}$ Andrews, D. U.; Heazlewood, B. R.; Maccarone, A. T.; Conroy, T.; Payne, R. J.; Jordan, M. J. T.; Kable, S. H. Science 2012, 337, 1203.

${ }^{8}$ Clubb, A. E.; Jordan, M. J. T.; Kable, S. H. J. Phys. Chem. Lett. 2012, 3, 3522.

${ }^{9}$ da Silva, G. Angew. Chem. Int. Ed. 2010, 49, 7523.

${ }^{10}$ da Silva, G.; Bozzelli, J. W. Chem. Phys. Lett. 2009, 483, 25.

${ }^{11}$ Karton, A.; Martin, J. M. L. J. Chem. Phys. 2012, 136, 124114.

${ }^{12}$ Vereecken, L.; Francisco J. S. Chem. Soc. Rev. 2012, 41, 6259.

${ }^{13}$ Zhang, R.; Khalizov, A.; Wang, L.; Hu, M.; Xu, W. Chem. Rev. 2012, 112, 1957.

${ }^{14}$ Zhang, R. Science 2010, 328, 1366.

${ }^{15}$ Nadykto, A. B.; Yu, F. Chem. Phys. Lett. 2007, 435, 14. 
${ }^{16}$ Hou, G.-L.; Lin, W.; Deng, S. H. M.; Zhang, J.; Zheng, W.-J.; Paesani, F.; Wang, X.-B. J. Phys. Chem. Lett. 2013, $4,779$.

${ }^{17}$ Martin, J. M. L.; Oliveira, G. J. Chem. Phys. 1999, 111, 1843.

${ }^{18}$ Peterson, K. A.; Feller, D.; Dixon, D. A. Theor. Chem. Acc. 2012, 131, 1079.

${ }^{19}$ Helgaker, T.; Klopper, W.; Tew, D. P. Mol. Phys. 2008, 106, 2107.

${ }^{20}$ Karton, A.; Daon, S.; Martin, J. M. L. Chem. Phys. Lett. 2011, 510, 165.

${ }^{21}$ Martin, J. M. L. J. Mol. Struct. (THEOCHEM) 2006, 771, 19.

${ }^{22}$ Karton, A.; Parthiban, S.; Martin, J. M. L. J. Phys. Chem. A 2009, 113, 4802.

${ }^{23}$ Karton, A.; Kaminker, I.; Martin, J. M. L. J. Phys. Chem. A 2009, 113, 7610.

${ }^{24}$ Stephens, P. J.; Devlin, F. J.; Chabalowski, C. F.; Frisch, M. J. J. Phys. Chem. 1994, 98 11623.

${ }^{25}$ Grimme, S.; Ehrlich, S.; Goerigk, L. J. Comput. Chem. 2011, 32, 1456.

${ }^{26}$ Dunning, T. H. J. Chem. Phys. 1989, 90, 1007.

${ }^{27}$ Kendall, R. A.; Dunning, T. H.; Harrison, R. J. J. Chem. Phys. 1992, 96, 6796.

${ }^{28}$ Dunning, T. H.; Peterson, K. A.; Wilson, A. K. J. Chem. Phys. 2001, 114, 9244.

${ }^{29}$ Gonzalez, C.; Schlegel, H. B. J. Chem. Phys. 1989, 90, 2154-2161.

${ }^{30}$ Frisch, M. J.; Trucks, G. W.; Schlegel, H. B.; Scuseria, G. E.; Robb, M. A.; Cheeseman, J. R.; Scalmani, G.; Barone, V.; Mennucci, B.; Petersson, G. A.; et al. Gaussian 09, Revision D.01, Gaussian, Inc., Wallingford CT, 2009. See also: http://www.gaussian.com.

${ }^{31}$ Werner, H.-J.; Knowles, P. J.; Manby, F. R.; Schütz, M.; Celani, P.; Knizia, G.; Korona, T.; Lindh, R.; Mitrushenkov, A.; Rauhut, G.; et al. Molpro 2012.1; University College Cardiff Consultants Limited: Cardiff U.K., 2012. See also: http:www.molpro.net.

${ }^{32}$ Peterson, K. A.; Feller, D.; Dixon, D. A. Theor. Chem. Acc. 2012, 131, 1079.

${ }^{33}$ Hattig, C.; Klopper, W.; Kohn, A.; Tew, D. P. Chem. Rev. 2012, 112, 4.

${ }^{34}$ Peterson, K. A.; Adler, T. B.; Werner, H.-J. J. Chem. Phys. 2008, 128, 084102.

${ }^{35}$ Hill, J. G.; Peterson, K. A.; Knizia, G.; Werner, H.-J. J. Chem. Phys. 2009, 131, 194105.

${ }^{36}$ Ten-no, S.; Noga, J. WIREs Comput. Mol. Sci. 2012, 2, 114.

${ }^{37}$ Knizia, G.; Werner, H.-J. J. Chem. Phys. 2008, 128, 154103.

${ }^{38}$ Knizia, G.; Adler, T. B.; Werner, H.-J. J. Chem. Phys. 2009, 130, 054104.

${ }^{39}$ Peterson, K. A.; Dunning, T. H. J. Chem. Phys. 2002, 117, 10548.

${ }^{40}$ Douglas. M.; Kroll, N. M.; Ann. Phys. 1974, 82, 89.

${ }^{41}$ B. A. Hess, Phys. Rev. A 1986, 33, 3742.

${ }^{42}$ de Jong, W. A.; Harrison, R. J.; Dixon, D. A. J. Chem. Phys. 2001, 114, 48. 
${ }^{43}$ Vöhringer-Martinez, E.; Hansmann, B.; Hernandez, H.; Francisco, J. S.; Troe, J.; Abel, B. Science 2007, 315, 497.

${ }^{44}$ Karton, A.; O’Reilly, R. J.; Radom, L. J. Phys. Chem. A 2012, 116, 4211.

${ }^{45}$ Karton, A.; O’Reilly, R. J.; Chan, B.; Radom, L. J. Chem. Theory Comput. 2012, 8, 3128.

${ }^{46}$ Curtiss, L. A.; Redfern, P. C.; Raghavachari, K.; Pople, J. A. J. Chem. Phys. 2001, 114, 108.

${ }^{47}$ Curtiss, L. A.; Redfern, P. C.; Raghavachari, K. WIREs Comput. Mol. Sci. 2011, 1, 810.

${ }^{48}$ Zheng, J.; Zhao, Y.; Truhlar, D. G. J. Chem. Theory Comput. 2009, 5, 808.

${ }^{49}$ Curtiss, L. A.; Redfern, P. C.; Raghavachari, K. Chem. Phys. Lett. 2010, 499, 168. 
TOC Graphic 1

1

2

3

4

5

6

7

8

9

10

11

12

13

14

15

16

17

18

19

20

21

22

23

24

25

26

27

28

29

30

31

32

33

34

35

36

37

38

39

40

41

42

43

44

45

46

47

48

49

50

51

52

53

54

55

56

57

58

59

60

61

62

63

64

65

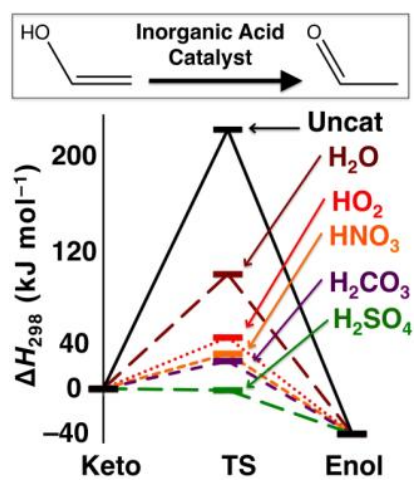




\author{
Click here to download Supplementary Materials: InorgAcidsTropo_CPL_SI_rev.docx
}

Click here to download Supplementary Materials: InorgAcidsTropo_cPL_Sl_rev.docx

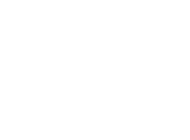

(1)

$\sqrt{3}$

(1)

(1)

.

(1)

.

.

.

.

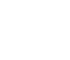

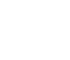

.

.

.

.

.

.

.

.

.

.

.

.

.

.

.

.

.

.

.

.

.

.

.

.

. 